\title{
KTR I HOCHELASTISCHE FLANSCHKUPPLUNGEN IM XXL-FORMAT
}

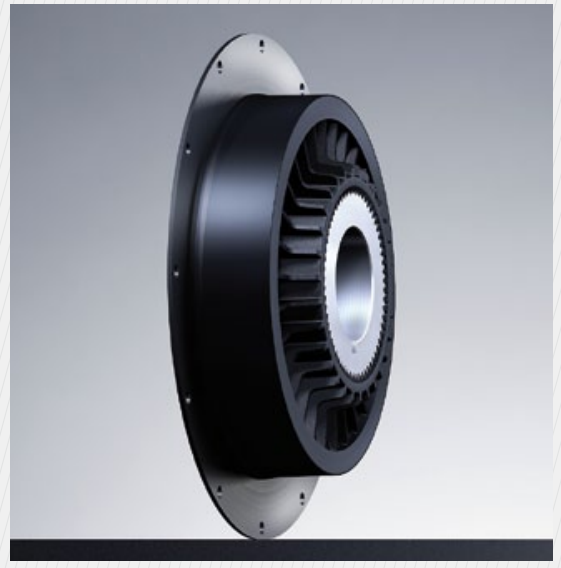

\begin{abstract}
Mit der BoWex-Elastic HE hat KTR eine Baureihe von hochelastischen Flanschkupplungen entwickelt, die nicht nur das anliegende Drehmoment zuverlässig überträgt, sondern auch Fluchtungsfehler im verbrennungsmotorischen Antriebsstrang zuverlässig ausgleicht und Schwingungen im Antriebssystem wirkungsvoll dämpft. Die Kupplungen bewähren sich bereits in der Praxis in Baumaschinen und anderen mobilen Arbeitsmaschinen. Jetzt wird die Baureihe um drei weitere Baugrößen FÜR DREHMOMENTE VON BIS ZU 20.000 NM ergänzt. Die Kupplungsnaben basieren auf der bekannten und bewährten BoWex-Bogenverzahnung, die eine gute Verlagerungsfähigkeit mit hoher Lebensdauer bietet. Die Flanschabmessungen der neuen Baugrößen orientieren sich an den üblichen SAEAbmessungen. Zu den weiteren Vorteilen der Kupplungen gehört die axiale Steckbarkeit, die eine einfache Montage gewährleistet.
\end{abstract}

\section{HYUNDAI HEAVY INDUSTRIES I NEUER KURZHECK-MINIBAGGER}

Zwischen den Hyundai-Minibaggern R16-9 und dem R27Z-9 schließt der zur bauma 2013 vorgestellte Minibagger R25Z-9A eine wichtige Lücke in der 2,6-T-KLASSE der Mini-Kettenbagger. Der von einem Mitsubishi-Motor (Tier 4) angetriebene Minibagger mit Z-Bezeichnung ( $Z$ = Zero-Turn-Radius, also eine Maschine, die innerhalb ihrer Konturen schwenkt und damit extrem platzsparend arbeitet) kann mit Tieflöffeln bis $0,07 \mathrm{~m}^{3}$ bestückt werden. Für den europäischen Markt wird der Minibagger mit verglaster ROPS-/ FOPS-Kabine angeboten. Die Kabine bietet mit einem gefederten Sitz, klappbaren Pedalen, feinfühligen Joysticks und verschiedenen Ablageflächen sehr ergonomischen Fahrerkomfort. Der neue Bagger ist $4030 \mathrm{~mm}$ lang, $2500 \mathrm{~mm}$ hoch und $1500 \mathrm{~mm}$ breit. Serienmäßig ist er mit 250-mmGummiketten für besonders niedrigen Bodendruck und Planierschild ausgerüstet - mit zahlreichen optionalen Zusatzausstattungen lässt er sich aber auch für Einsätze im Hoch-, Tief- und Galabau anpassen.

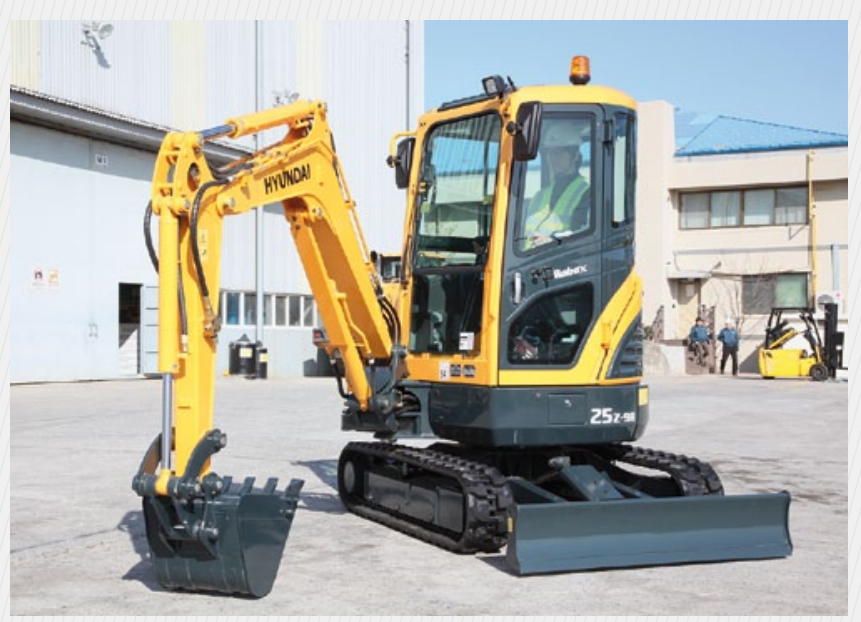

\section{IVECO I OFFROAD-BAUSTELLENFAHRZEUG}

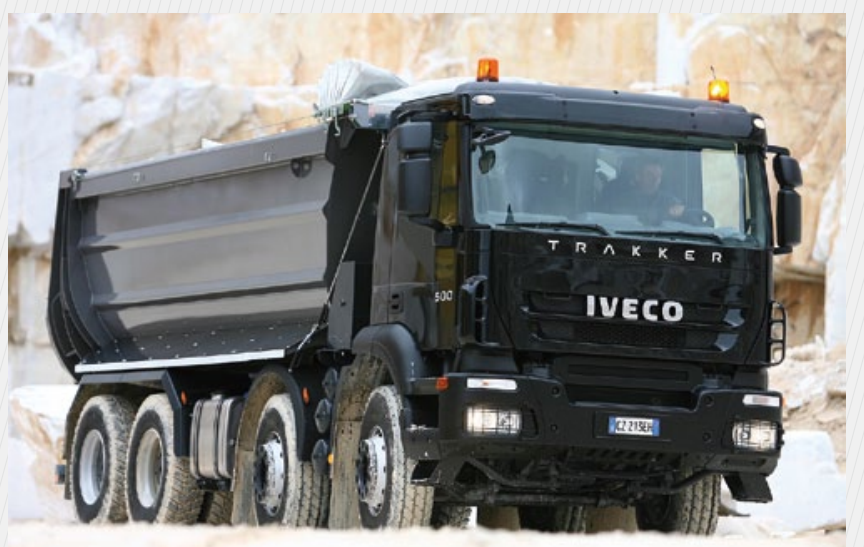

Iveco präsentiert als Ausstellungsschwerpunkt auf der bauma den neuen Trakker, ein Offroad-Baustellenfahrzeug mit NEU GESTALTETEM FAHRERHAUS. Das Baustellenfahrzeug ist dank der Cursor-Motoren (Cursor 8 und Cursor 13) ideal für raues, unebenes Gelände. Der Cursor 8 hält die EEVStandards mühelos ein, die noch strenger als die Euro-V-Vorschriften sind. Der Cursor 13 ist gemacht für anspruchsvolle Einsätze auf schwierigem Gelände und unter widrigen Bedingungen. Er liefert sein hohes Drehmoment bereits bei niedrigen Drehzahlen und sorgt so für ein entspanntes Fahrgefühl. Der Cursor 13 ist mit 331 oder 368 kW mit VTG-Lader beziehungsweise $302 \mathrm{~kW}$ mit normalem Turbolader und Wastegate erhältlich. 